\title{
Magnetic Effects in the Thermodynamics of Information
}

Aibassov Erkin Zhakenovich ${ }^{* 1}$, Yemelyanova Valentina Stepanovna ${ }^{1}$, Shakieva Tatyana ${ }^{1}$, Tussupbaev Nessipbay ${ }^{1}$, Savizky Ruben ${ }^{2}$, Bulenbayev Maxat ${ }^{1}$ and Blagikh Evgeniy ${ }^{1}$

1. Research Institute of New Chemical Technologies and Materials, Kazakh National University Al-Farabi, Almaty 005012, Kazakhstan

2. Department of Chemistry, the Columbia University, NewYork 005012, USA

Abstract: The authors have shown that the process data can be modeled on the basis of chemical thermodynamics. The authors offer general information of the equation in the presence of a magnetic field. As a result, studies have shown that the magnetic effects strongly influence the thermodynamics of information.

Key words: Magnetic effect, thermodynamics, information, Shonon equation.

\section{Introduction}

The human brain is the most mysterious organ that controls thought processes.

In recent years, many works devoted to the thermodynamics of information [1-6].

The authors have previously been shown that the thermodynamic functions of internal energy $d U$ and free energy $d F$ in the presence of a magnetic field [5]:

$$
\begin{gathered}
d U=T d S+1 / 4 \pi H d B \\
d F=-S d T+1 / 4 \pi H d B
\end{gathered}
$$

It was also shown that the Nernst equation in a magnetic field change, and is described by the Eq. (3) [6]:

$$
E=E^{0}+R T / n F \ln a_{O x} / a_{R e d}+1 / 4 \pi H d B
$$

The purpose of this work is an attempt to study the magnetic effects in the thermodynamics of information processes.

\section{Theory}

The information in contrast to the thinking can not appear as a product of pure inferences from other data. It is impossible, without spending any work, just standing on the platform, by "pure" reasoning to know address of your friend.

\footnotetext{
*Corresponding author: Aibassov Erkin Zhakenovich, professor, research fields: metalorganic chemistry of uranium and thorium, As, Sb and Bi.
}

Information data are logically independent of each other, it is as if from nowhere output set of "primary", independent data (in physical chemistry—-the system of non-interacting particles).

Entropy - a measure disorder and uncertainty of any state of the system.

If the information and intellectual activity is indeed connected with the atomic-molecular mechanisms, then they should be subject to all the laws of thermodynamics, and in particular, the law of conservation of energy and the law of increasing entropy.

The main function of information theory—Shannon function

$$
H_{\text {Шен }}=-\Sigma p_{i} \log p_{i},
$$

is defined as "information entropy", that is how it really undetectable and has such properties.

Considering the zero state of information system bezentropiynoe state $\left(H_{0}=0\right)$, then every state is different from zero, is equal to the Shannon entropy:

$$
\Delta H=H-H_{0}=H_{\text {Шен, }}
$$

If all the outcomes are equally likely, it is a system of Z-multiple of "degenerate”, the information entropy of such a system will be:

$$
H_{\text {Max }}=\log \mathrm{Z} \text {, }
$$

In order to make information about some of the facts necessary costs of work for the destruction of the 
information entropy. This is the amount of information $I$, equal to the Shannon entropy with the opposite sign:

$$
I=-H_{\text {Шен }}
$$

Entropy-one of the complex concepts of thermodynamics, which often puts students in a deadlock. The processes taking place without additional supply of energy from outside (constant-energy processes), a decrease of internal energy of the system is accompanied by a proportional increase in entropy and vice versa. In all known physical processes spontaneous entropy tends to increase, and this fact was the reason for a deeper analysis of entropy. This analysis was carried out in the last century by Boltzmann. It turns out that the entropy expresses the probability of a physical system to be in this state.

In any physical, chemical or biological processes are involved atoms and molecules. In every moment of their thermal motion which creates a situation (state) is not similar to the one that was a second ago. So, entropy is a measure of the transition of the body or bodies from less probable to a more probable state, from less stable state to a more stable. This entropy increases.

The increase in entropy is not something mysterious. It follows directly from the atomic and molecular structure of all bodies in the universe, as well as atoms and molecules are always in motion, they tend to scatter throughout the infinite space.

If the entropy as a measure of probability of a physical system, and its growth is the transition from the smaller to the larger order, the authors can make some generalizations. The most important of them appeared when there was information theory.

Information theory, like thermodynamics, enters as a measure of the amount of information associated with the probability judgments about the system, and also calls this the entropy.

It is not only in a formal analogy. The relationship between entropy and information system, that is the knowledge of her condition, much deeper.

Imagine a vessel filled with hydrogen. With this volume of gas can be done a number of experiments to determine its temperature, pressure and point out that all the atoms undergo thermal motion in a given region of space. Thus, the authors can get some information about the considered gas.

What happens if the vessel is opened to allow hydrogen atoms to blend with the surrounding air? The entropy of the gas will start to grow steadily, and our knowledge of it every second will be increasingly uncertain until the authors fully not "lose sight of him”, that is, cease to be aware of it something concrete!

It turns out that entropy decreases with increasing information on the system!

In order to lead the two notions to one form, the information measured in the same way as the entropy, but only with the sign "minus".

Similarly, the thermodynamic entropy to reduce the degree of ignorance about the system also needs to do the work. It's called work information.

\section{Results and Discussion}

The human mind is the repository of a variety of information, derived as a result of experience or through training. This information and people enjoy every moment throughout his adult life. He needs it to perform a wide variety of work tasks. Every second, he draws from this giant store the necessary information as to "forget" about the huge amount of other information that he may need in other aspects of his life.

Extracting specific information from consciousness, it lowers the entropy of the entire system, making thus the work is exactly equal to the decrease in the initial entropy.

Consciousness, in which information is stored, you can imagine in the form of a volume where information is distributed in a certain way ("recorded" in the molecules of ribonucleic acid). Before such time as 
willed man chosen out of all the storage is quite certain, desired him at this point the information, its position resembles the familiar situation, the address of which the authors know nothing. After making the work a person retrieves information from the consciousness he needed data that corresponds to the transition of the system in a completely unambiguous state, when the mind is fixed on only one "Exodus" (the exact address).

Selection of the required information is reduced to the translation of "chance particles" in one, and the total volume of compression to the value that uniquely corresponds to the desired information.

Job information that must be expended to transform uncertain system definitions can write equation:

$$
\begin{aligned}
I^{o}=I_{o}^{o}-A & =-\log Z+\left(\Sigma p_{i} \log p_{i}+\log Z\right) \\
& =\Sigma p_{i} \log p_{i}=I_{\text {Vin }}
\end{aligned}
$$

Since reversible isothermal work is a decrease in entropy, then

$$
\Delta H=-\Sigma p_{i} \log p_{i}=H_{\text {Sonnon }}
$$

The calculation of the thermodynamic work on such a "chance-gas" leads to a value that corresponds exactly to the data calculated by the American scientists Shannon and Wiener on the basis of the general theory of information.

The authors have proposed that the presence of a magnetic field, the Eqs. (7) and (8) are transformed into:

$$
\begin{gathered}
I^{o}=I_{o}^{o}-A+1 / 4 \pi H d B=\Sigma p_{i} \log p_{i}= \\
I_{V i n}+1 / 4 \pi H d B \\
\Delta H=-\Sigma p_{i} \log p_{i}+1 / 4 \pi H d B= \\
H_{\text {Sonnon }}+1 / 4 \pi H d B
\end{gathered}
$$

Information modeling in the form of an ideal "chance-gas" leads to conclusions that are consistent with the theory of information, and it follows that all the information processes may be performed on the atomic and molecular level.

\section{Conclusion}

The analysis shows that the information is modeled on the basis of chemical thermodynamics in the form of spontaneous transition (Z-1) varieties of "chances" that are concentrated in a single cell in a k-th some sort of a drop in the free energy and entropy, expressed by the equation of thought.

The authors offer general equations to calculate the information in the presence of a magnetic field:

$$
\begin{gathered}
I^{o}=I_{o}^{o}-A+1 / 4 \pi H d B=\Sigma p_{i} \log p_{i}= \\
I_{\text {Vin }}+1 / 4 \pi H d B \\
\Delta H=-\Sigma p_{i} \log p_{i}+1 / 4 \pi H d B= \\
H_{\text {Sonnon }}+1 / 4 \pi H d B
\end{gathered}
$$

The authors have shown that the information can be modeled on the basis of chemical thermodynamics. The authors offer general equations to calculate the information in the presence of a magnetic field. As a result, studies have shown that the magnetic effects strongly influence the thermodynamics of the process of information.

The consequence of the above is the ability to save the information entropy and speed of thinking in three dimensions, so the speed of thought to be increased by several orders of magnitude.

Research in this important for the study of the process of obtaining, storing and processing information in that direction the authors will continue.

Transmission of thought at a distance, the influence of magnetic fields on the behavior and mental condition, "clairvoyance", "premonition" and many other psychiatric manifestations that hitherto could not be explained by known us physicochemical mechanisms may be interpreted in a new way. And here it is not so much to prove or disprove the existence of these and other mysterious phenomena, but in the development of completely new ways of approach to the objective study of mental.

\section{Acknowledgments}

The authors would like to thank Lynn C. Francesconi (Hunter College CUNY), Peter C. Burns (Notre Dame University, Indiana) and Chistopher L. Cahill (George Washington University) for discussion of the results. 


\section{References}

[1] Claude, E. 1948. "Shannon: A Mathematical Theory of Communication.” Bell System Technical Journal 27: 379-423.

[2] Claude, E. S., and Warren, W. 1949. The Mathematical Theory of Communication. The University of Illinois Press, Urbana, Illinois.

[3] Claude, E. S. 1950. Programming a Computer for
Playing Chess, Philosophical Magazine, Ser. 7, 41: 314.

[4] David, L. 1983. Computer Gamesmanship: Elements of Intelligent Game Design. Simon \& Schuster.

[5] Aibassov, Y., and Yemelyanova, V. 2014. "Study of Changes in the Thermodynamic Functions in a Magnetic Field.” J. Chem. Chem. Eng. 8: 119-22.

[6] Aibassov, Y., and Yemelyanova, V. 2015. "Derivation of the Equation Nernst-Aibassov in a Magnetic Field.” $J$. Chem. Chem. Eng. 9: 218-220. 